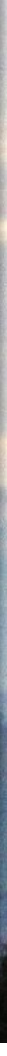

\title{
El Campo Volcánico Xalapa: una revisión y análisis
}

Katrin Sieron - Fátima Carreto - Oliver McLeod - Marie Noelle Guilbaud - Francisco Córdoba Montiel - Juan Cervantes Pérez

RESUMEN: El Campo Volcánico Xalapa (CVX) consta de 42 conos de escoria y al menos 2 volcanes escudos, cuyas edades varían entre 2 millones de años (M.a.) hasta 870 años (a) antes del presente (datos radiométricos publicados). Mediante la determinación de los parámetros morfométricos, se estimaron las edades relativas de los conos de escoria (tipo de volcán predominante en el CVX) aun no fechados. La densidad de los conos de escoria dentro del campo varia de 0 hasta 2 por km2. El uso de la función kernel permite identificar zonas con mayor densidad de volcanes al NW de Xalapa entre Acajete y Las Vigas de Ramírez (grupo La Joya) y al norte de Coacoatzintla, mientras zonas con concentraciones menores están localizadas alrededor de Pacho Viejo y al norte de Ayahualulco. Aunque el Campo Volcánico Xalapa no es el campo con mayor número de edificios del Cinturón Volcánico Trans-Mexicano (CVTM), ni el más denso; sin embargo se debe crear consciencia de que puede dar lugar al nacimiento de un nuevo volcán en el futuro. Un monitoreo sería deseable.
ABSTRACT: The Xalapa Monogenetic Volcanic Field (XMVF) defines a group of 42 scoria cones and at least two lava shields that erupted between 2 million and 870 years before present (according to published radiometric data). In this study, we measured the morphometric parameters of XMVF scoria cones in order to assign relative ages to undated volcanic centers. The density of vents within the limits of the volcanic field (estimated by using spatial point density analysis) and found to vary from 0 to a maximum of 2 volcanoes per square kilometer. Kernel density function analysis revealed that the highest concentrations of volcanoes occur NW of Xalapa between Acajete and Las Vigas de Ramirez (La Joya group), and $\mathrm{N}$ of Coacoatzintla, while lesser concentrations occur around Pacho Viejo and to the N of Ayahualulco. Although the XMVF is relatively small and has a low vent-density compared to other volcanic fields in the Trans-Mexican Volcanic Belt, a greater level of public awareness and hazard monitoring, is required in preparation for the next XMVF eruption. 
Introducción

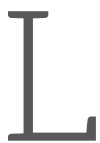
a parte central de México esta atravesada del Este al Oeste ( $E$ al W) por una franja con volcanes activos, así como dormidos o extintos, y sus productos. El volcanismo a lo largo de esta franja denominada Cinturón Volcánico Transmexicano (CVTM), inició en el Terciario (Mioceno; p.ej. Ferrari et al., 2012) y tiene su origen en el proceso de la subducción de las placas tectónicas Rivera y Cocos (ambas oceánicas) por debajo de la placa continental de Norteamérica (Figura 1). Durante este proceso, agua y otros componentes móviles se liberan de las placas subducidas, bajando así la temperatura del punto de fusión del manto, lo que a su vez lleva a la producción de magma (explicado de una manera muy simplificada). Los volcanes en sí son la expresión en la superficie de este proceso; a través de zonas con debilidad cortical emiten productos que se acumulan alrededor de la fuente.

En México y en el mundo, la investigación científica así como la atención publica se concentra en los volcanes que están activos, por ejemplo en el Popocatépetl cerca de las ciudades de México y Puebla, o el Colima, cerca de la ciudad con el mismo nombre, ambos con muestras de su actividad en estos últimos años. Estos volcanes grandes y visibles a largas dis- tancias, son en su mayoría del tipo estratovolcán (edificios altos, cónicos, longevos que reciben su nombre por la alternancia de productos piroclásticos como cenizas y lavas).

En consecuencia, en la consciencia común, no están "presentes" los miles de volcanes en el país que se originan en grupos, caracterizándose por ser edificios de pequeñas dimensiones, $y$ por haber tenido una sola erupción (por ello se denominan "monogenéticos"). El más joven de estos volcanes es el Paricutin (Michoacán) que nació en 1943 y mostró actividad por más de 9 años (p.ej. Luhr y Simkin, 1993). Es poco probable que uno de estos volcanes vuelva a "despertar", pero una gran parte de los volcanes en el CVTM son geológicamente jóvenes (Holoceno y Pleistoceno tardío) y nuevos volcanes se pueden formar en distintas áreas propensas a lo largo del CVTM.

Estos volcanes ocurren generalmente en grupos denominados "campos monogenéticos", de los cuales existen varios en el país con un número de decenas hasta cientos de pequeños edificios volcánicos. Hay tres tipos de volcanes monogenéticos, de los cuales los conos de escoria (p.ej. Paricutín) son los más comunes, seguidos por pequeños volcanes escudo cráteres de explosión y domos de lava.

Los volcanes monogenéticos cada vez reciben más atención, aunque todavía no la misma

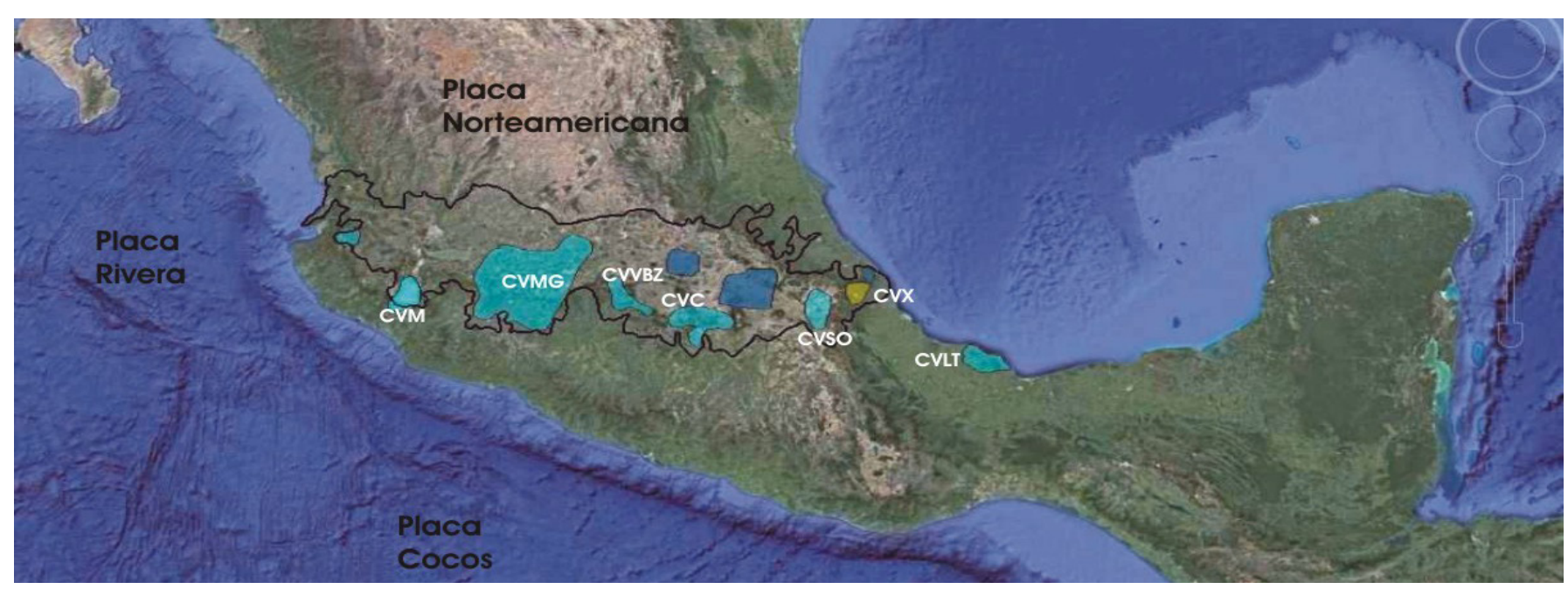

Figura 1. Imagen Google Earth modificada. Se muestran las placas tectónicas Cocos y Rivera que se subducen por debajo de la Norteamericana, así como el Cinturón Volcánico Mexicano con una línea negra. Se indican algunos Campos monogenéticos del CVTM. Del E al W: CVM=Campo Volcánico Mascota, CVMG=Campo Volcánico Michoacán Guanajuato, CVVBZ=Campo Volcánico Valle de Bravo, CVC= Campo Volcánico Chichinautzin, CVSO=Campo Volcánico Serdán Oriental, CVX=Campo Volcánico Xalapa, CVLT= Campo Volcánico Los Tuxtlas. Los campos con color azul (Jilotepec, Apan/Tezontepec y Chiconquiaco del W al E), son más antiguos que los verdes (y el CVX en amarillo). 
que sus parientes de dimensiones más importantes.

En el CVTM, del W al E, están los campos volcánicos monogenéticos de Colima-Cántaro (Luhr y Carmichael, 1981), San Pedro-Ceboruco (Petrone, 2010; Petrone et al., 2006), Mascota (Ownby et al, 2008), Michoacán-Guanajuato (Hasenaka y Carmichael, 1985), Chichinautzin (Bloomfield, 1975; Martin, 1982; Siebe et al., 2004, 2005), Apan-Tezontepec (García-Palomo et al., 2002; Carrasco-Núñez et al., 2005), Campo Valle de Bravo (Jaimes-Viera, 2004), Serdán-Oriental (Riggs y Carrasco-Núñez, 2004; Carrasco-Núñez et al., 2007), Chiconquiaco-Palma Sola (López-Infanzón, 1991; Siebert y Carrasco-Núñez, 2002; Ferrari et al., 2005) y el Campo Volcánico Los Tuxtlas (Nelson y González-Caver, 1992).

El grupo de volcanes monogenéticos alrededor de la ciudad Xalapa ha tenido nombres y extensiones diferentes; aunque en estudios recientes se le ha denominado Campo Volcánico Xalapa (González-Mercado, 2005; Rodríguez et al., 2010). En estudios anteriores se refieren en parte a esta región como Naolinco-Jalapa (Negendank et al., 1985), Cofre de Perote y campo volcánico Naolinco (Siebert y Carrasco-Núñez, 2002).

En este estudio, a partir de la disponibilidad de modelos topográficos de alta resolución (Lidar) y en el marco de los proyectos (PRODEP e INEGI) y la realización de una tesis de Licenciatura, se visitaron los edificios del Campo Volcánico Xalapa para estudiar su morfología, se tomaron muestras para fechamiento de sus productos y se analizó su distribución espacial usando métodos estadísticos.

\section{Metodología}

Según los trabajos más recientes anteriores sobre el Campo Volcánico Xalapa (Rodríguez et al. (2010), este campo se integra por 59 volcanes. En este estudio, los volcanes mencionados por Rodríguez et al. (2010) se ubicaron y delimitaron en imágenes satelitales, orthofotos, y modelos de elevación. De estos 59, se seleccionaron los 21 conos de escoria mejor preserva- dos para estudios morfométricos utilizando los Sistemas de Información Geográfica (SIG) con imágenes Lidar (INEGI, 2012), modelos de elevación generados a partir de la información topográfica de INEGI para las zonas sin cobertura por Lidar, así como imágenes de Google Earth.

Usando la superficie digital así creada, se pudieron delimitar con precisión las bases y cráteres de los conos de escoria para luego medir los parámetros morfométricos individuales con las herramientas del SIG, específicamente: Wco (diámetro promedio del cono), Wcr (diámetro promedio del cráter), Hco (altura promedio del cono) de acuerdo con Porter (1972) y Wood (1980). La pendiente S (pendiente promedia) se calculó mediante la fórmula

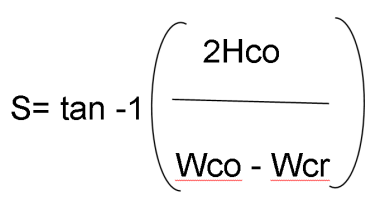

(Hasenaka y Carmichael, 1985). Para la medición del volumen también se siguió al trabajo de Hasenaka y Carmichael (1985) utilizando la fórmula establecida para el cálculo del volumen de un cono truncado simétrico: $V=\pi \mathrm{H} / 12$ (W2cr + Wcr Wco + W2co).

Se llevó a cabo la verificación en campo de los 59 volcanes para comprobar el tipo de volcán y sus parámetros estimados. Además se observaron el estado de preservación, el tipo de uso de suelo y la existencia de minas de grava, estructuras de erosión, etc. Tal como mencionado arriba, sólo 21 conos de escoria aplicaron para llevar a cabo el análisis morfométrico completo, dado que los demás han sido demasiado afectados por procesos naturales como la erosión o por el uso del humano (extracción de material en graveras) para que los parámetros morfométricos puedan ser establecidos de manera confiable. Los trabajos pioneros de Porter (1972) y Wood (1980a, 1980b) relacionan parámetros morfométricos sencillos de conos de escoria (altura y diámetro de la base) con su edad relativa, con la premisa que un cono de escoria evoluciona morfológicamente de manera sistemática con el paso del tiempo (típicamente la altura del cono disminuye mientras su base 
se ensancha). También, recientemente se han usado métodos cada vez más sofisticados para derivar tales parámetros (p.ej. Inbar et al. 2011; Kereszturi et al. 2012).

Los 21 conos se agruparon en 3 grupos según ciertos rangos de edades relativas estimadas con las relaciones de los parámetros morfométricos, como Hco/Wco y Wcr/Wco de acuerdo con Porter (1972), Wood (1980) y Hasenaka y Carmichael (1985).

Los mapas de densidad de los conos de escoria del CVMX se elaboraron a partir de herramientas de los SIG, como es la "densidad de puntos", para determinar cuántos conos se ubican por km2, y la densidad kernel para identificar las áreas más activas en el pasado (es decir con mayor número de volcanes).

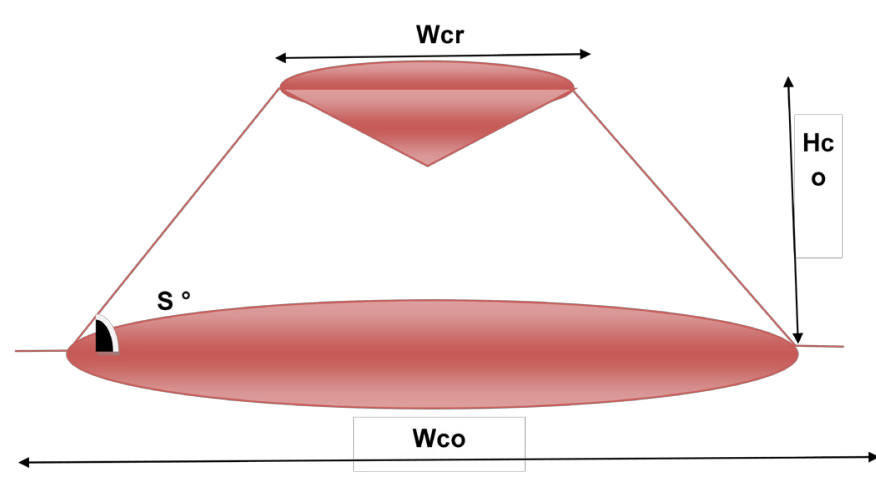

Figura 2. Esquema de un cono de escoria ideal. La figura muestra los parámetros morfométricos incluidos en análisis morfométrico de conos cineríticos. Wco (diámetro de la base), Wcr (diámetro del cráter), Hco (altura del cono) y S (pendiente de laderas externas) (modificado de Wood, 1980).

\section{El Campo Volcánico Xalapa 2.1 Geología}

El basamento antiguo, previo al inicio del volcanismo, consiste de rocas calcáreas del Mesozoico que forman parte de la topografía montañosa de la Sierra Madre Oriental, así como algunos complejos de rocas plutónicas y metamórficas cerca del Cofre de Perote (Yáñez y Garcia, 1982). Sobreyaciendo este basamento, afloran secuencias clásticas del Terciario que se extienden hasta la cuenca de Veracruz.
El vulcanismo inició con la emisión de lavas fisurales al norte del campo (Chiconquiaco) con las unidades más antiguas fechadas con entre 6 y 7 millones de años (Ma) (Ferrari et al., 2005; López-Infanzón, 1991), sobreyacidos por otros flujos de lava que afloran cerca de Actopan con edades alrededor de $2 \mathrm{Ma}$ (Ferrari et al., 2005).

El CVX en sí fue descrito por primera vez por González-Mercado (2005), refiriéndose a una secuencia de rocas volcánicas formadas a partir de volcanes monogenéticos distribuidos en los alrededores de la Ciudad Xalapa. Este autor reporta el análisis morfométrico de 20 volcanes monogenéticos clasificados en cuatro tipos de estructuras (conos de escoria, conos de lava, volcán tipo escudo y anillos piroclásticos), que juntos abarcan una superficie de 905 km2. Rodríguez et al. (2010) consideran que dentro de un área de unos 2,400 km2 en la parte central del estado de Veracruz, alrededor de Xalapa, se encuentran más de 50 volcanes monogenéticos. La mayoría de ellos son conos de escoria, en menor proporción pequeños volcanes escudo y domos de lava.

Estudios previos del CVX indican que las edades de los volcanes y sus productos oscilan alrededor del Pleistoceno tardío-Holoceno (p. ej. Negendank, 1985; Siebert y Carrasco-Núñez, 2012; Rodríguez et al., 2010) con base en fechamientos de Argón/Argón, Potasio/Argón y radiocarbono. Los conos de escoria más antiguos del CVX son el Cerro Estropajo con 2.5 $\mathrm{Ma}$, Cerro Colorado 3 con 2.2 Ma, La Malinche con 2.1 (Rodríguez et al., 2010); aparte de los volcanes Las Lajas 1 y 2 y Cerro Roma con 5 a 7 Ma (según el Servicio Geológico Mexicano) y Pacho Viejo con alrededor de $2 \mathrm{Ma}$ (Rodríguez et al., 2010). Una edad similar (2.2 Ma, Rodríguez et al., 2010) también tienen los depósitos de ignimbrita El Castillo que cubren gran parte del CVX.

Posteriormente inicia la actividad en el área del Cofre de Perote (CP) (fase 1 del CP; 1.3-0.51 $\mathrm{Ma}$ ) con flujos y domos de lava. Una lava en la cercanía del Acatlán fue fechada en 1.2 Ma (López-Infanzón, 1991), pero el cono tiene un aspecto morfológico mucho más joven. Una segunda ignimbrita (Xaltipan) fue emitida desde Los Humeros alrededor de 0.42 Ma (Ferriz 
y Mahood, 1984). Lavas masivas se emitieron desde el CP, representando la fase principal de la construcción del escudo; también nacieron dos conos de escoria con edades similares (Zimpizahua con 0.39 y Acamalín con 0.29 Ma, Rodríguez et al., 2010). La última fase del CP se concentró en la cima con flujos de lava evolucionados (0.25 Ma). Al sur de Xalapa nació Las Lomas (0.25 Ma, Siebert y Carrasco-Nuñez, 2002) y al Norte Cerro Mocho (0.19 Ma, Rodríguez et al., 2010). El Cerro Macuiltépetl se fechó con $0.08 \mathrm{Ma}$ (Rodríguez et al., 2010) y emitió primero un gran escudo de lava (gran parte del área urbanizada de Xalapa) y un cono de escoria en la cima.

Al Norte, La Joya 1 nació alrededor de 40,000 años BP (Siebert y Carrasco-Núñez, 2002). Las lavas asociadas al grupo La Joya se emplazaron al SE hasta encontrarse con el escudo del Macuitépetl. Los demás conos del grupo La Joya son más jóvenes que 40,000 años, pero más viejos que 3000 años BP (Siebert y Carrasco-Núñez, 2002), edad del flujo de lava Coatzintla rodeando La Joya 4.
CP tuvo en segundo evento de colapso alrededor de 11-13,000 BP (depósitos Xico). Al norte de Xalapa se formó Rincón de Chapultepec (2980 a AP=fecha del flujo de lava emitido).

La actividad volcánica más reciente ocurrió en el Volcancillo, el cual produjo dos flujos de lava muy extensos: el flujo de lava Toxtlacuaya que fluyó hacia el complejo La Joya (edad de 910 años AP, longitud de $12 \mathrm{~km}$ ) (Siebert y Carrasco-Núñez, 2002) y el flujo Río Naolinco que viajó hasta $50 \mathrm{~km}$ de su fuente y es ligeramente más joven que Toxtlacuaya.

\subsection{Integrantes del Campo Volcánico Xalapa}

Los volcanes monogenéticos tienen una cierta morfología que, en el caso de tratarse de un edifico geológicamente "joven", es fácilmente identificable en mapas topográficos e imágenes satelitales. Sin embargo, un análisis solamente basado en imágenes conlleva una elevada tasa de error, ya que los procesos de erosión pueden producir "geoformas" que se asemejan a la for-

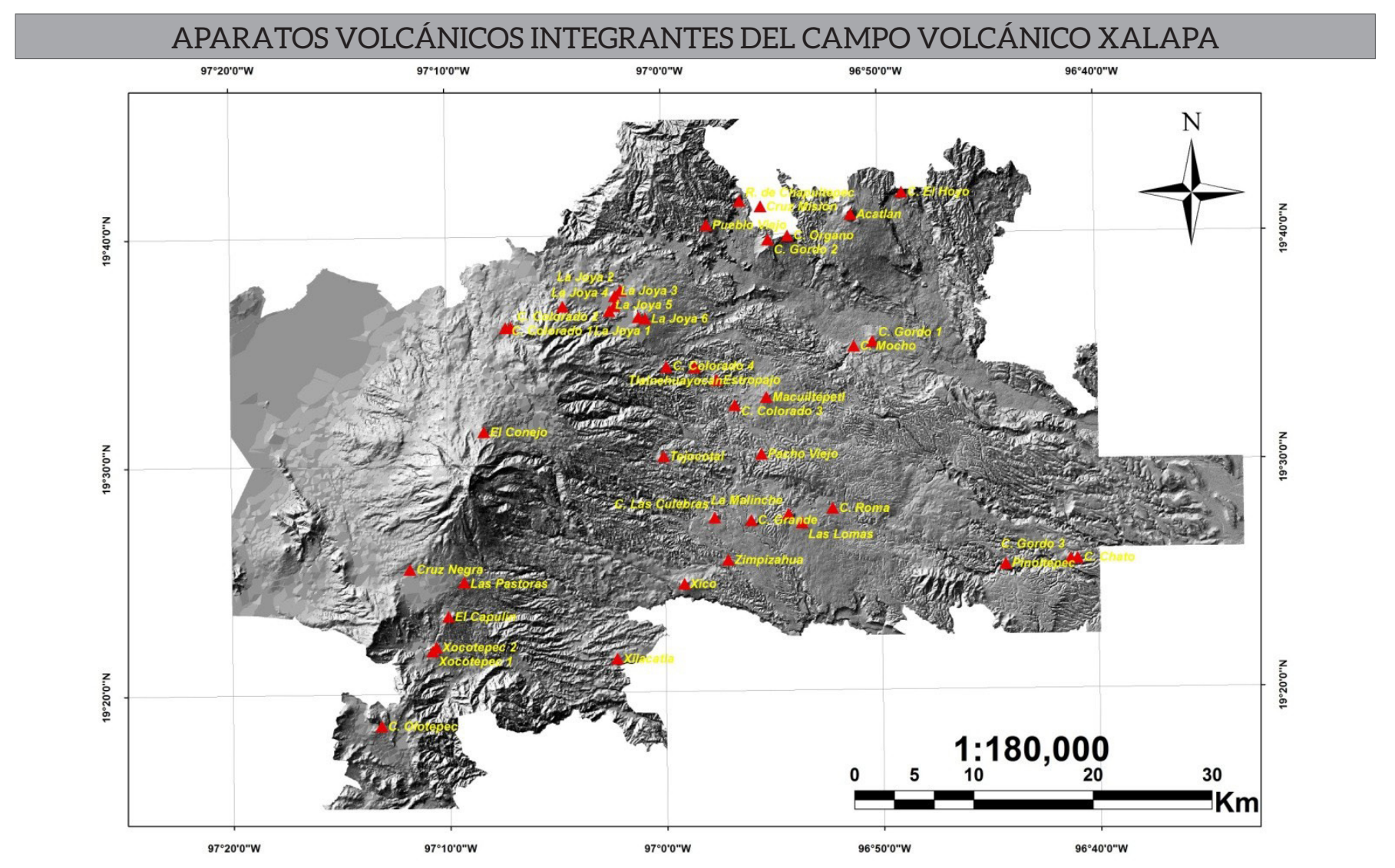

Figura 3. Modelo Digital de Elevación (sombreado) mostrando los resultados del análisis de los integrantes del CVX, después de la revisión bibliográfica y la posterior revisión (uno por uno) en campo (elaboración propia). 
ma de un cono de escoria, así como es posible de encontrarse con volcanes erosionados que difícilmente se reconocerían como un cono de escoria. Por ello, después de múltiples salidas al campo, se han descartado varios integrantes reportados por los autores anteriores (como ejemplo quisiéramos mencionar el Cerro la Pedrera que representa una formación de calizas, que en forma de mina están siendo extraídos). También se agregaron nuevos conos de escoria que se han encontrado en campo por encontrar los llamativos depósitos (grava roja, negra o gris) en campo.

\subsection{Morfometría}

EEn el área de estudio, el diámetro basal de los conos de escoria (Wco) presenta un rango de $116 \mathrm{~m}$ a $1160 \mathrm{~m}$ con una media de $435 \mathrm{~m}$ y una mediana de $437 \mathrm{~m}$. Los valores del diámetro del cráter (Wcr) se encuentran entre 43 m y 445 m, la media es de $184 \mathrm{~m}$ y la mediana de $200 \mathrm{~m}$. La altura (Hco) media de los conos de escoria es de $68 \mathrm{~m}$ y la mediana de $60 \mathrm{~m}$, los valores oscilan entre 20 m y 210 m; la pendiente por otro lado presenta un valor máximo de casi $50^{\circ}$ y un mínimo de $16^{\circ}$, la media para este parámetro es de $29^{\circ}$ y la mediana de 31 . El rango de volumen de los conos de escoria de la zona de estudio oscila entre 0.0023 km3 y 0.009 km3, los valores de la media y la mediana son 0.0121 km3 y 0.0152 km3, respectivamente, tienen alturas más bajas que las establecidas para un cono de escoria de altura máxima de $300 \mathrm{~m}$.

Analizando la variabilidad de cada uno de los parámetros morfométricos, se pueden observar (Figura 3) que seis conos de escoria poseen un diámetro basal (Wco) entre 400 m y 500 m y cuatro conos entre 300 a 400 m, cuatro conos entre los 500 a 700 m y otros cuatro de los 100 a 300 m, el resto se distribuye entre los 700 m a 1200 m (Figura 4A). Respecto al diámetro del cráter (Wcr), la mayoría de los conos se ubican en un intervalo de 100 a 300 m (Figura 4B). Los conos poseen una altura (Hco) entre $20 \mathrm{~m}$ y $210 \mathrm{~m}$ y la mayoría de ellos se encuentra en un intervalo de 51-100 (Figura 4C), mientras que la pendiente está entre $15.8^{\circ}$ y $210^{\circ}$ (Figura 4D). Los volúmenes de la mayoría de los conos de escoria se encuentran entre los valores de 0.0023 y $0.009 \mathrm{~km} 3$, seis conos muestran valores entre 0.041 y 0.0701 , y entre 0.0152 y 0.0248 se encuentran cuatro conos de escoria, el resto se distribuye entre los 0.0355 -0.002 km3 (Figura 4E). Los valores del diámetro basal como del diámetro del cráter son diferentes a los reportados por Rodríguez et al. (2010). Por otra parte en el caso de los datos obtenidos por González Mercado, también difieren los datos de las mediciones morfométricas. Los conos que coinciden en su estudio pre-

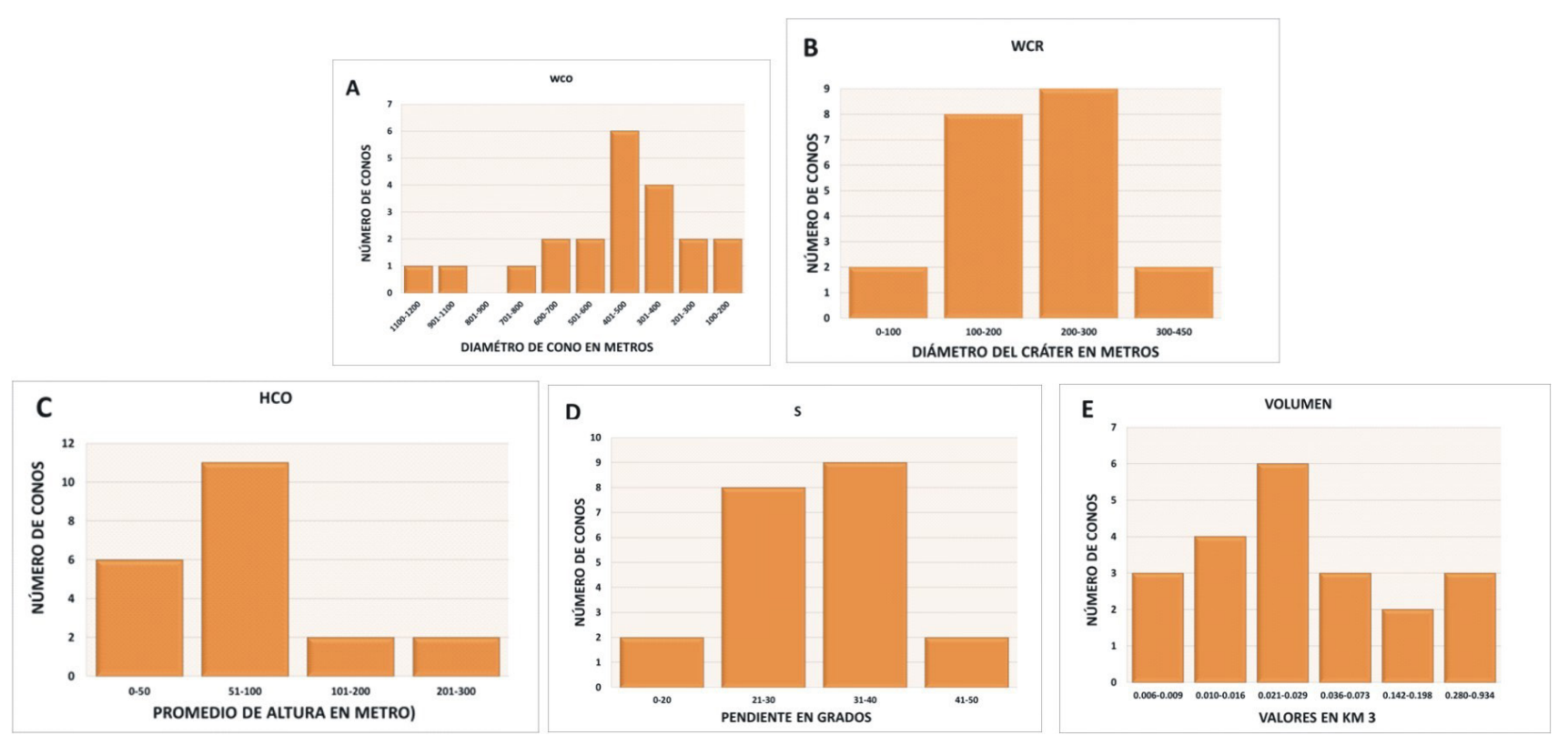

Figura 4..Histogramas representando un resumen de las variaciones de los parámetros morfométricos en el CVX (Wco/diámetro de la base, Wcr/diámetro del cráter, Hco/altura del cono y S/pendiente de laderas externas). 
vio con este nuevo análisis morfométrico son el Macuiltépetl, Cerro Gordo 1, Las Culebras, Cerro Grande, y Las Lomas. La diversidad de mediciones de los mismos parámetros muestra que hay variaciones considerables según la metodología empleada, la calidad de las imágenes utilizadas, así como la experiencia del colector de los datos.

2.4 Edades relativas de los volcanes integrantes del Campo

Con base en el trabajo anterior de Siebert y Carrasco (2002) en el CVX, en este estudio se optó por una clasificación en tres grupos de edades. A diferencia del trabajo anterior se indicaron rangos de edades tomando en cuenta todos los conos de escoria ya fechados (ver cap 3), asi como los parámetros morfométricos y rasgos morfológicos de los conos sin fechar (ver Figura 5 y Tabla 1). Como resultado se definen tres grupos (A, B y C) con rangos de edades probables de $<10,000$ años para el grupo de conos de escoria más joven, 10,000 a 0.7 M.a. para el intermedio y de 0.7 a 2.2 M.a. para el grupo con los conos más antiguos

\section{Peligro asociado}

En resumen de lo anterior, la verificación de los trabajos previos tanto en imágenes satelitales como en el campo permitió confirmar la naturaleza de 45 de los 52 volcanes identificados por Rodríguez et al. (2010).

Analizando la densidad de edificios dentro del campo se puede observar (Figura 6), que por 5 kilómetros cuadrados existen de $\mathrm{O}$ a 3 volcanes.

Este valor de hasta 3 volcanes por $5 \mathrm{~km} 2$ (o de entre 0 y 2 volcanes por km2) es un valor promedio a bajo, comparando con otros campos volcánicos, como por ejemplo Los Tuxtlas con hasta 6 volcanes por km2 en las zonas más densas (Sieron et al., 2014) y Michoacán Guanajuato con hasta 9 volcanes por km2.

Para mejor visualización de las áreas mayormente pobladas por conos de escoria, se realizó una estimación de densidad usando la función

\section{GRUPO DE EDADES GEOLÓGICAS DE LOS CONOS DE ESCORIA}

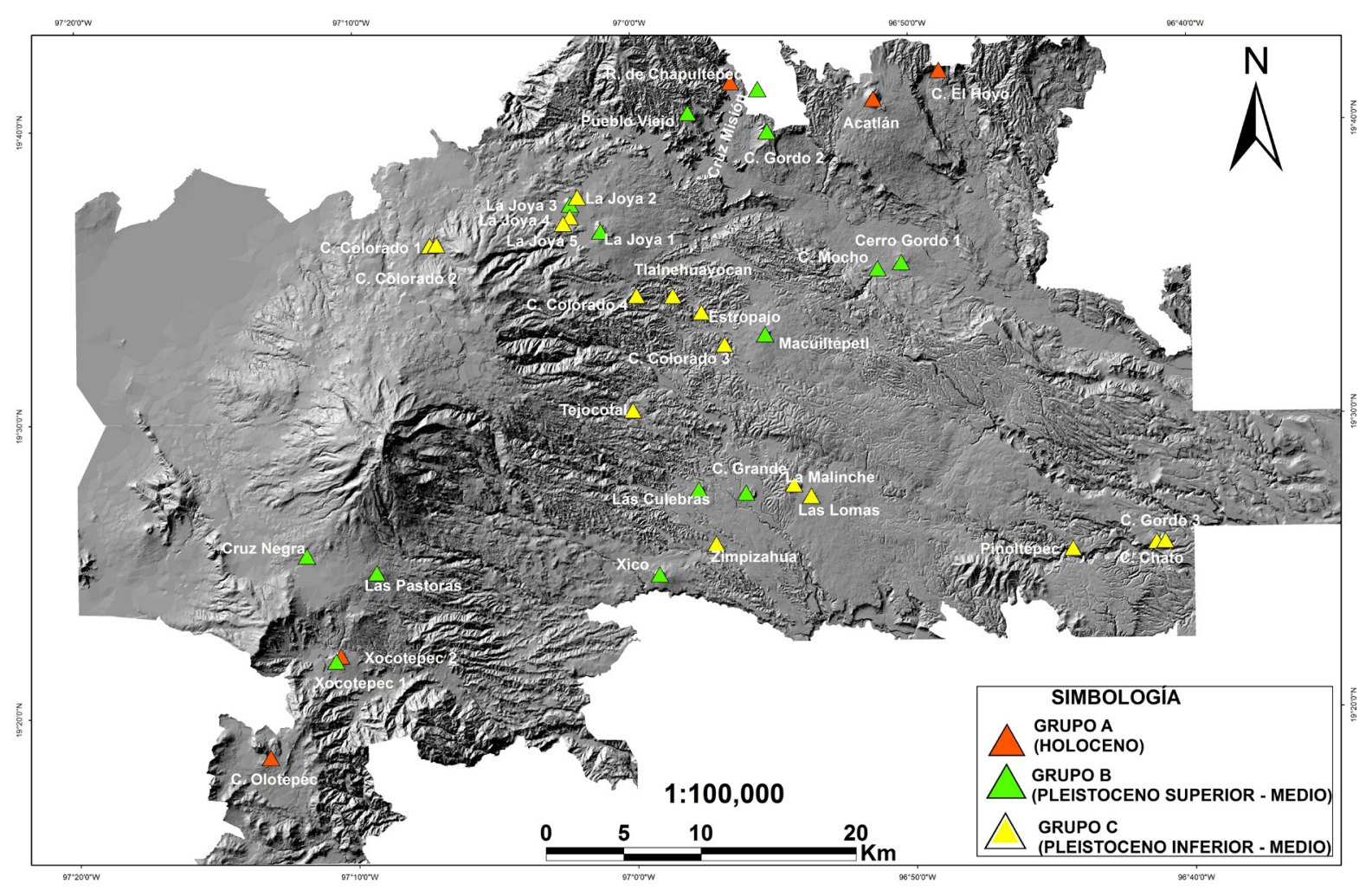

Figura 5. Edades relativas de los volcanes integrantes del CVX. 
Tabla 1. Edades relativas según el presente estudio de los volcanes del CVX de acuerdo con los criterios de Porter (1972), Wood (1980) y Siebert y Carrasco (2002).

\begin{tabular}{|c|c|c|}
\hline $\begin{array}{c}\text { GRUPO A } \\
\text { HOLOCENO } \\
<10,000 \text { a }\end{array}$ & $\begin{array}{c}\text { GRUPO B } \\
\text { PLEISTOCENO SUPERIOR } \\
\text { MEDIO } \\
\text { 0,781 M.a. -10000 a }\end{array}$ & $\begin{array}{c}\text { GRUPO C } \\
\text { PLEISTOCENO INFERIOR } \\
\text { 2,558 - 0,781 M.a }\end{array}$ \\
\hline $\begin{array}{l}\text { Hco/Wco } 0.15-0.27 \\
\text { Pendiente }>30^{\circ}\end{array}$ & $\begin{array}{c}\text { Hco/ Wco } 0.13-0.14 \\
\text { Pendiente } 23-29^{\circ}\end{array}$ & $\begin{array}{l}\mathrm{Hco} / \text { Wco } 0.10-0.13 \\
\text { Pendiente }<22^{\circ}\end{array}$ \\
\hline Acatlán & Acamalin & La Joya 2 \\
\hline C. Hoyo & C. Gordo 2 & La Joya 4 \\
\hline Rincón Chapultepec & C. Molino & La Joya 5 \\
\hline Olotepec & La Joya 1 & C. Las Lomas \\
\hline \multirow[t]{12}{*}{ Xocotepec 2} & La Joya 3 & Tejocotal \\
\hline & C. Las Culebras & Malinche \\
\hline & Pueblo Viejo & Tlalnehuayocan \\
\hline & Xocotepec 1 & C. Gordo 3 \\
\hline & C. Grande & Zimpizahua \\
\hline & C.Gordo 1 & Pinoltepec \\
\hline & Macuiltépetl & Estropajo \\
\hline & C. Mocho & C. Colorado 3 \\
\hline & Cruz Misión & C. Colorado 4 \\
\hline & Cruz Negra & C. Chato \\
\hline & & C. Colorado 1 \\
\hline & & C. Colorado 2 \\
\hline
\end{tabular}

kernel del SIG (Figura 7). Las zonas donde más se concentran los volcanes se aprecian en la parte norte, noroeste y sur-este del CVX que corresponden a las áreas de Naolinco, La Joya, Coatepec y Ayahualulco-Perote.

Estas áreas de mayor densidad de volcanes monogenéticos también son más susceptibles a presentar nuevas formaciones de volcanes en el futuro; en especial cerca de Naolinco y Ayahualulco, ya que son las zonas donde se registraron los conos de escoria jovenes, lo que sugiere una zona volcánicamente activa. Esta idea se basa en que las zonas donde hayan ocurrido más erupciones en el pasado son propensas a sufrir un nuevo ascenso del magma por las caracteristicas de la corteza (por ejemplo, corteza delgada o fracturada); por lo consecuente es válido asumir que tambien en el futuro estas zonas presentan una mayor probabilidad de erupciones, por lo que la función kernel ha sido utilizada para interpretarse como función de probabilidad (p. ej. Connor et al., 2012).

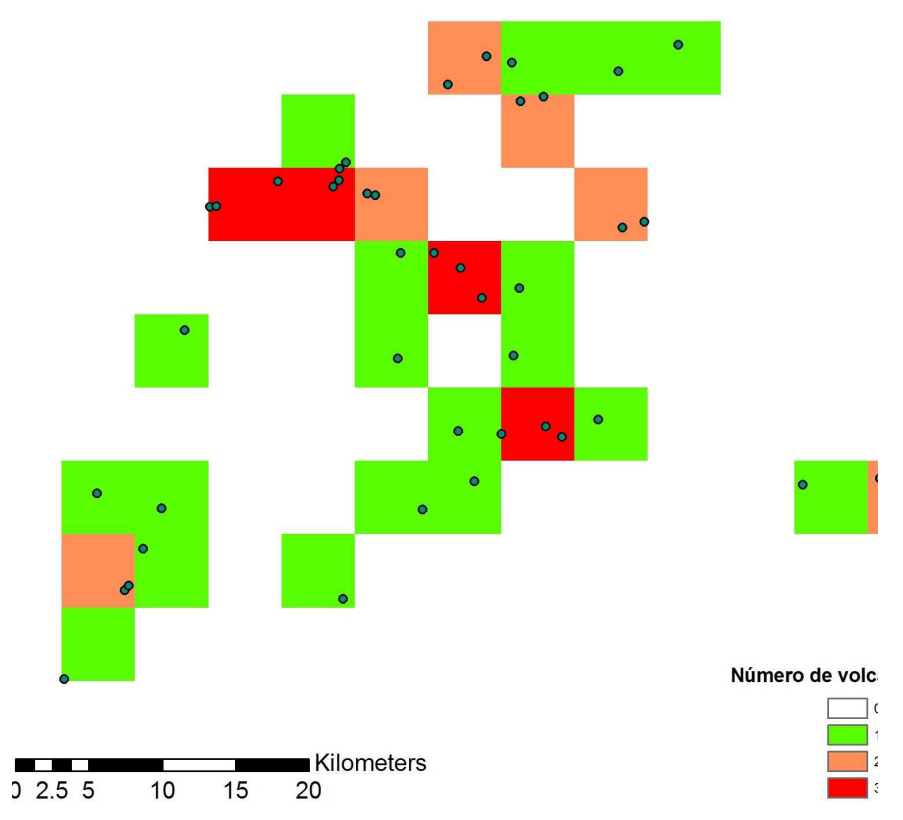

Figura 6. Densidad de volcanes en el CVX por kilómetro cuadrado. 


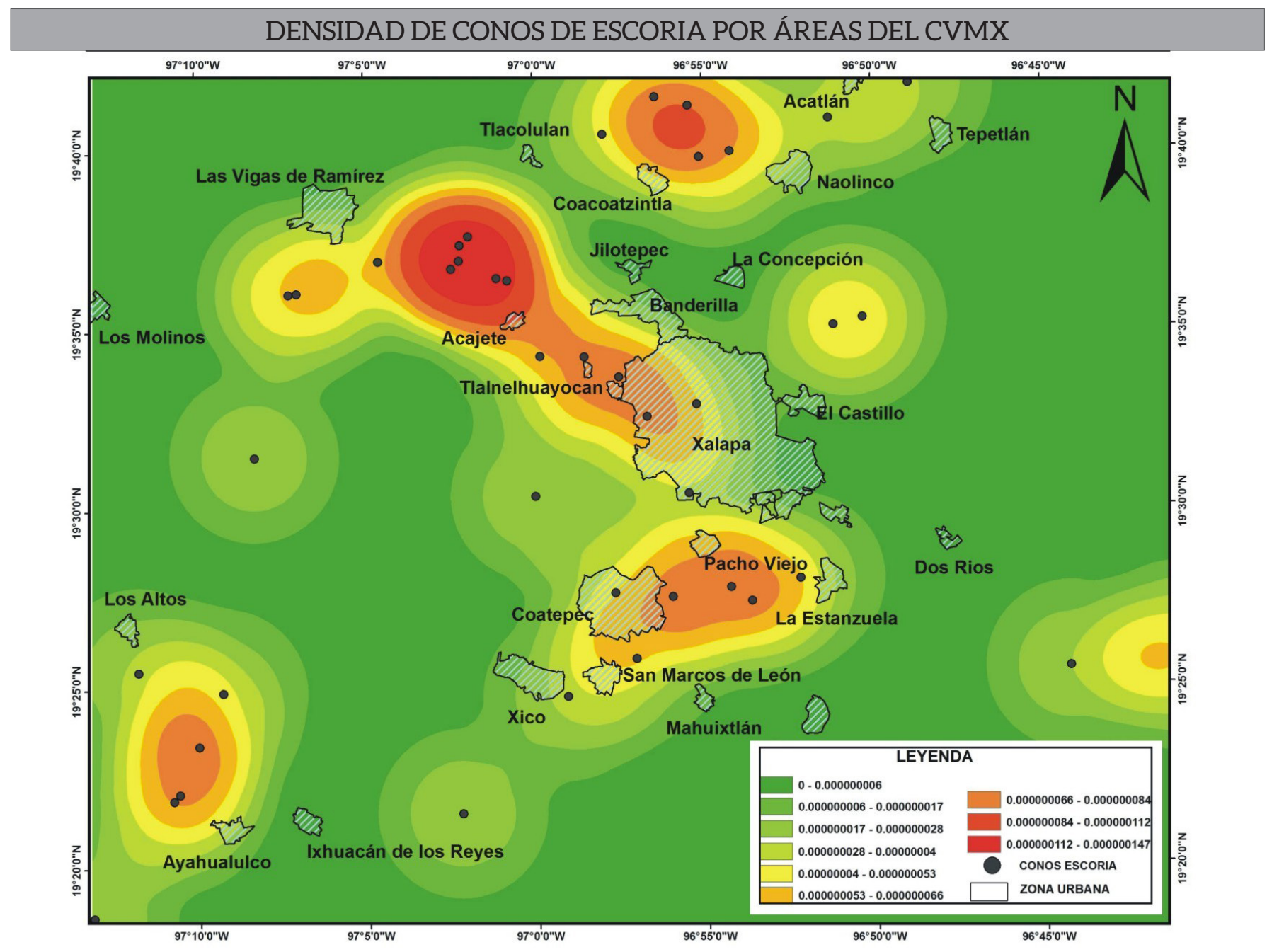

Figura 7. Densidad de volcanes en el CVX determinados mediante la función kernel (SIG, elaboración propia).

\section{Conclusiones}

En este estudio se determinó, que los 59 aparatos de escoria formando el CVX (Rodríguez et al 2010) incluyen elevaciones que no son conos de escoria, disminuyendo el número total de volcanes del CVX por 14; los descartados incluyen formaciones de caliza e ignimbrita, así como formaciones de lava considerablemente más antiguas que el campo mismo. Esto también lleva a la conclusión, que el trabajo minucioso en campo es indispensable en este tipo de estudios, ya que hasta los insumos más modernos (como son las imágenes Lidar) no ofrecen la información para identificar volcanes sin error. También se debe mencionar que existen discrepancias considerables en la medición de los parámetros morfométricos de un mismo volcán en diferentes trabajos, por lo que sería deseable de unificar metodologías matemáticas para comparar de manera objetiva las morfologías de este tipo de volcanes.

Mediante el estudio de los parámetros morfométricos (apoyado por fechamientos ya existentes de algunos volcanes) y morfológicos se pudo clasificar los conos de escoria en viejos, intermedios y jóvenes. El Campo Volcánico Xalapa existe desde los 2.0 Ma aproximadamente y desde entonces el paisaje volcánico ha sido modificado; además de los cambios continuos y lentos, provocados por los procesos endógenos (p.ej. nacimiento de nuevos volcanes) y exógenos (procesos de erosión), también se observan cambios drásticos que ocurren dentro de un lapso corto de tiempo con origen en procesos antropogénicos (por ejemplo la extracción de material).

La futura actividad volcánica dentro del CVX (erupciones similar a lo que se presenció en el nacimiento del volcán Paricutin) probablemen- 
te afectaría a las poblaciones de Acajete, Coacoatzintla, Naolinco, Coatepec y Ayahualulco por encontrarse cerca (distancia $<2 \mathrm{~km}$ ) a los grupos de conos de escoria que presentan la mayor densidad.

Es importante mencionar que en la actualidad se monitorean en México una variedad de los volcanes "grandes" (estratovolcanes) considerados activos, también tarea de este Observatorio Sismológico y Vulcanológicoa. El monitoreo generalmente consiste en la observación de sismicidad, y adicionalmente de la deformación en el edificio volcánico, el análisis de emisiones de gas y fuentes hidrotermales cercanas al volcán. Esto permite de "anticipar" una erupción dentro de un cierto margen de tiempo muy variable. Sin embargo, en el caso de los volcanes monogenéticos, que son el resultado de un pulso magmático único y cuyos magmas no residen por tiempos alargados en la corteza antes de ascender y entrar en erupción (cortos tiempos de sismicidad anticipada), además de nacer en lugares impredecibles, un monitoreo es difícil (se necesita cubrir un área extensa con una densa red de sensores y aun así persisten la problemáticas mencionadas) por lo que representan un reto a futuro.

Otro reto es crear consciencia de que Xalapa está inmersa en una región volcánica y que hay que estar preparados para el caso de una eventual actividad futura.

\section{Referencias}

Bloomfield, K. 1975, A Late-Quaternary monogenetic volcano field in central México.Geol. Rundsch.64, 476497.

Carrasco-Nuñez, G., Righter, K., Chesley, J., Siebert, L., Aranda-Gómez, J.G. (2005). Contemporaneous eruption of cal-alkaline and alkaline lavas in continental arc. Eastern Mexican Volcanic Belt. Chemically heterogeneous but isotopically homogeneous source. pp 423-440.

Carrasco-Núñez, G.Ort, MichaelH., Romero, C., 2007. Evolution and hydrological conditions of a mar volcano (Atexcac cráter, Eastern Mexico). J. Volcanol. Geotherm. Res.pp 179-197.

Connor y L.J. Connor, 2012, Estimating spatial density with kernel methods in. En C.B. Connor, N.A Chapman, y L.J. Connor editors, Volcanic and Tectonic Hazard Assessment for Nuclear Facilities. University Press.pp 331343.

Ferrari, $\quad$ L., $\quad$ Tagami,T.,Eguchi,M.,Orozco-Esquivel,M.T.,Petrone,C.M.,Jacobo-Albarrán, J., López-Martínez, M. 2005. Geology, geochronology and tectonic setting of late Cenozoic volcanism along the southwestern Gulf of Mexico: the Eastern Alkaline Province revisited.J. Volcanol. Geotherm. Res. Pp 24-306.

Ferrari L, Orozco-Esquivel M.T., Manea V, Manea M. 2012. The dynamic history of the Trans-Mexican Volcanic Belt and the Mexico subduction zone. Tectonophysics, Invited review paper, doi:10.1016/j.tecto.2011.09.018.

García-Palomo, A., Macias, J.L., Tolson, G., Valdez, G., Mora., J.C. (2002). Volcanic stratigraphy and geological evolution of the Apan región east-central sector of the Trans-Mexican Volcanic Belt. Geofis. pp 133-150.

González Mercado, D.E. (2005). El vulcanismo monogenético de la región de Xalapa, Veracruz. Geomorfología, petrología y génesis. Tesis de maestría. Instituto de Geología. UNAM.

Hasenaka, T. Carmichael, I. 1985. The cinder cones of Michoacán-Guanajuato, central México: their age, volumen and distribution, and magma discharge rate. J.Volcanol. Geotherm.Res. 25. pp 104-124.

Inbar, M., Gilichinsky, M., Melekestsev, I., Melnikov, D. and Zaretskaya, N., 2011.Morphometric and morphological development of Holocene cinder cones: A field and remote sensing study in the Tolbachik volcanic field, Kamchatka Journal of Volcanology and Geothermal Research, 201(1-4): 301-311

Jaimes-Viera, M.C. (2004). Geología y Geomorfología del Campo Volcánico Valle de Bravo. Undergraduate's thesis, Facultad de Ingeniería, Universidad Nacional Autónoma de México. Pp 95.

Kereszturi, G.; Jordan, G; Németh, K, and Dóniz-Páez, J.F. 2012. Syn-eruptive morphometric variability of monogeneti scoria cones. Bull. Volcanol. 74 (9): 2171-2185

López-Infanzón, 1991; López-Infanzón, M. (1991). Petrologic study of the volcanic rocks in the Chiconquiaco-Palma Sola área central Veracruz, México. Master's, Graduate School, Tulane University.pp 139.

Luhr y Carmichael, 1981 Luhr, J.F., Carmichael, I. (1981). The Colima Volcanic Complex, México. Late-Quaternary cinder cones. Contrib. Mineral. Petrol.

Luhr, J.F., Simkin, T. (eds) 1993, Paricutin. The volcano born in a Mexican cornfield. Geoscience Press, Inc., Arizona, USA, $427 \mathrm{p}$. 
Martin del Pozzo, A.L. 1982, Monogenetic volcanismin Sierra Chichinautzin México. Bull. Volcanol. pp 9-24

Negendank, J., Emmermann, R., Krawczyk, R., Mooser, F., Tobschal, H., Werles, D. (1985). Geological and geochemical investigations on the Eastern Trans-Mexican Volcanic Belt. Ed. Mexican Volcanic Belt Special Volume, part 2. Geofisica International, vol. 24.pp.477-575.

Nelson, S.A., González-Caver, E. (1992). Geology and k-Ar dating of the Tuxtla Volcanic Field, Veracruz, Mexico.Bull.Volcanol. pp 85-96.

Ownby, S.E., Lange, R.A., Hall, C.M. (2008). The eruptive history of the Mascota volcanic field, western México: age and volumen constraints on the origin of andesite among a diverse suite of lamprophyric and calc-alkaline lavas. J. Volcanol. Geotherm. Res.pp 1077-1091.

Petrone, C.M., 2010, Relationship between monogenetic magmaticm and stratovolcanoes in western Mexico: the role of low-pressure magmatic processes. Lithos, 119, pp. 585-606.

Petrone, C.M., Francalanci, L., Ferrari, L., Schaaf, P., Conticelli, S., 2006. The San-Pedro-Cerro Grande volcanic complex (Nayarit, Mexico): Inferences on volcanology and magma evolution. En: Siebe, C., Macías, J.L., Aguirre-Díaz, G.J. (Ed): Neogene-Quaternary continental margin volcanism: A perspective from Mexico. Geol. Soc. Am. Spec. Paper 402, 65-98.

Porter (1972) Porter, S. (1972).Distribution, morphology and size of cinder cones on Mauna Kea volcano, Hawaii. Geplogical Society of América Bulletin.

Riggs, N., Carrasco-Nuñez, G. (2004). Evolution of a complex, isolated dome system Cerro Pizarro, central Mexico.Bull.Volcanol. pp 322-335.

Rodríguez, S.R., Morales-Barrera, W., Layer., P., y González-Mercado., E. (2010). A quaternary nonogenetic volcanic field in the Xalapa región, Eastern Trans-Mexican volcanic belt: Geology, distribution and morphology of the volcanic vents. Journal of Volcanology and Geothermal Research, 197, 149-166.

Siebe, C., Arana-Salinas, L., Abrams, M., 2004. Radiocarbon ages of Holocene Pelado, Guespalapa, and Chichinauzin scoria cones, south of México City: implications for archeology and future hazards. Bull. Volcanol.pp 203-225.

Siebe, C., Arana-Salinas, L., Abrams, M. 2005. Geology and radiocarbon ages of Tlaloc, Tlacoteno, Cuauhtli, Hijo del Cuauhtli and Ocusacayo monogenetic volcanoes in the central part of Sierra Chichinautzin. Mexico. JVGR, 141, 225-243
Siebert, L., Carrasco-Núñez, G. (2002). Late-Pleistocene to precolumbian behind-the-arcmafic volcanismin in the Eastern Mexican Volcanic Belt; implications for future hazards, J. Volcano. Geotherm.Res. pp 179-205.

Sieron, K., Capra, L., Rodríguez, S. 2014. Hazard assessment at San Martin Volcano based on geological record, modeling and spatial analysis. Natural Hazards, 70, (1) pp. 275-297.

Wood, C.A. 1980a. Morphometric analysis of cinder cone degradation. Journal of Volcanology and Geothermal Research. pp. 137-160.

Wood, C.A. 1980b. Morphometric evolution of cinder cones. Journal of Volcanology and Geothermal Research., 7 (3-4) pp. 387-413

Yáñez, C., Garcia, S., 1982: Exploración de la región geotérmica Los Humeros-Derrumbadas, Estados de Puebla y Veracruz, Mexico, D.F. CFE. 